\title{
A Low-Cost Terahertz Camera
}

\author{
François Blanchard ${ }^{1, *}$, Joel Edouard Nkeck ${ }^{1}$, Dominique Matte ${ }^{2}$, Riad Nechache ${ }^{1}$ \\ and David G. Cooke ${ }^{2}$ \\ 1 Département de génie électrique, École de Technologie Supérieure (ÉTS), Montréal, QC H3C 1K3, Canada; \\ joel-edouard.nkeck.1@ens.etsmtl.ca (J.E.N.); riad.nechache2@etsmtl.ca (R.N.) \\ 2 Physics department, McGill University, Montréal, QC H3A 2T8, Canada; \\ dominique.matte@mail.mcgill.ca (D.M.); cooke@physics.mcgill.ca (D.G.C.) \\ * Correspondence: francois.blanchard@etsmtl.ca
}

Received: 31 May 2019; Accepted: 19 June 2019; Published: 21 June 2019

Featured Application: Low cost THz array detector is highly anticipated to offer affordable industrial solutions. Nowadays with thermal sensors, infrared vision is highly available for the general public. Using same technology, our data confirms its extension to the THz range.

\begin{abstract}
Cost effective imaging is required for a wide range of scientific and engineering applications. For electromagnetic waves in the terahertz $(\mathrm{THz})$ frequency range, a key missing element that has prevented widespread applications in this spectral range is an inexpensive and efficient imaging device. In recent years, vanadium oxide based thermal sensors have rapidly entered the market for night vision capability. At the same time, sensors based on this technology have been applied to the $\mathrm{THz}$ domain, but with two orders of magnitude larger pricing range. Here we show that, with a simple modification, a commercially available thermal imaging camera can function as a $\mathrm{THz}$ imaging device. By comparing a commercially available THz camera and this low-cost device, we identify the main sensitivity difference is not attributed to anything intrinsic to the devices, but rather to the analog-to-digital converter and dynamic background subtraction capability. This demonstration of a low-cost THz camera may aid in the rapid development of affordable $\mathrm{THz}$ imaging solutions for industrial and scientific applications.
\end{abstract}

Keywords: thermography; terahertz; imaging; camera; sensing; low cost

\section{Introduction}

Terahertz (THz) imaging methods have considerably evolved over the past decade [1,2] driven by a wide range of highly anticipated applications [3-10]. Some of the promising THz imaging prospects are for non-destructive testing of materials [3-5], medical and pharmaceutical applications [6,7], art conservation [8], security screening [9], and food inspection [10]. All these applications still face the same challenges, reducing acquisition time, simplifying the routing optics, compactness, ease-of-use, reliability and low-cost in fabrication and operation. On top of these issues, a second challenge is the technological accreditation over established technologies, such as X-ray and ultrasound imaging. These two burdens are like "the chicken or the egg" causality dilemma; solving the first problem will help addressing the second one, or vice-versa.

For scientific applications of $\mathrm{THz}$ waves, a great wall has been eliminated when researchers were finally able to visualize the THz light emitted from a nonlinear optical generation process [11]. Especially using a $\mathrm{THz}$ camera based on an uncooled microbolometer array [12,13], Hirori et al. monitored the lithium niobate output crystal using the titled-pulse-front pumping (TPFP) scheme, and demonstrated the merit of seeing the spatial mode of the emitted THz beam [14]. This important insight on the spatial $\mathrm{THz}$ beam profile greatly helped in producing intense $\mathrm{THz}$ pulses at the focus 
position, which have opened several doors for nonlinear light-matter interactions in this frequency range [15]. However, the scientific market of $\mathrm{THz}$ radiation applications being limited, their related products, such as imaging devices, are generally unaffordable for a straightforward commercialization procedure. Basically, other walls need to fall before a $\mathrm{THz}$ industrial revolution.

Here we show a simple $\mathrm{THz}$ imaging demonstration using a widely available infrared camera for thermal imaging. As a light source, a broadband, single-cycle pulsed THz emitter based on the tilt-pulse front pumping (TPFP) scheme in lithium niobate crystal was employed, similar to the system used for THz pulse imaging demonstration using uncooled microbolometer array [14]. Our results indicate comparable sensitivities between a thermal imaging camera (Seek Thermal Company) and a scientific $\mathrm{THz}$ imaging camera (Institut National d'optique (INO) de Québec). The main drawback of the low-cost thermal imaging camera when combined with pulsed $\mathrm{THz}$ measurements is the lack of dynamic background subtraction and the low-resolution red, green, blue (RGB) saving format, all fairly extrinsic features that can easily be mitigated.

\section{Materials and Methods}

To perform our imaging comparison, a $\mathrm{THz}$ source based on the tilted-pulse-front pumping (TPFP) scheme in a lithium niobate crystal was employed, as illustrated in Figure 1a. The pumping source consists of an amplified Ti-Sapphire femtosecond laser providing $120 \mathrm{fs}$ duration, $5 \mathrm{~mJ}$ pulses at a repetition rate of $1 \mathrm{kHz}$ with a center wavelength of $800 \mathrm{~nm}$. As shown in Figure 1b, the THz source delivered intense $\mathrm{THz}$ pulses centered at $1 \mathrm{THz}$ with a peak electric field at the focus on the order of $500 \mathrm{kV} / \mathrm{cm}$. According to its approximately $3 \mu \mathrm{J}$ in energy per pulse, the maximum average $\mathrm{THz}$ power available is approximately $3 \mathrm{~mW}$. To perform our imaging demonstration, we used an uncooled microbolometer camera (IRXCAM-384THz from INO [13]) as a reference sensor and compared its response with a low-cost thermal camera (Compact pro from Seek Thermal). Both cameras were tested at the focus of the $\mathrm{THz}$ source, as illustrated in Figure 1a. Notice that to record images or movies using the Compact Pro camera, either an Apple iPhone or Android device is required. Both cameras have a similar number of pixels and are based on the sensing technology of vanadium oxide material $[13,16]$. The microbolometer array from INO is composed of $384 \times 288$ pixels with $35 \mu \mathrm{m}$ in pitch, whereas, the Compact Pro used an uncooled microbolometer array of $380 \times 240$ pixels with $12 \mu \mathrm{m}$ in pitch. Since vanadium oxide sensors are highly sensitive to ambient heat given their insulating to metal transition at $\approx 340 \mathrm{~K}$, a mechanical chopper is included inside the Compact pro camera. This shutter is activated depending on the background information. On the other hand, the IRXCAM- $384 \mathrm{THz}$ is easily triggered via a BNC connector, thus enabling synchronous background subtraction when combined with a mechanical chopper. This option radically improved the imaging contrast of $\mathrm{THz}$ acquisitions and is obviously desirable for accurate measurements.

In order to turn the Compact Pro thermal camera into a THz sensor, the small chalcogenide lens used to form images at the sensor position must be removed. Indeed, while highly suitable for the infrared wavelength range (i.e., from $1 \mu \mathrm{m}$ to $10 \mu \mathrm{m}$ ), the absorption coefficient of such material is extremely high in the THz frequency range [17]. Figure 2a shows the difference in absorption coefficients between a high resistivity silicon wafer and a typical chalcogenide glass material in the $\mathrm{THz}$ frequency range. The inset in Figure 2a shows a zoomed view of the small $0.02 \mathrm{~cm}^{-1}$ in power absorption of a high resistivity $(>20,000 \mathrm{ohm}-\mathrm{cm})$ silicon wafer, in good agreement with [18]. By removing the 7-mm-thick chalcogenide lens, the vanadium oxide sensor can be directly illuminated with $\mathrm{THz}$ radiation without suffering from the high attenuation factor of chalcogenide glass (e.g., $>80 \mathrm{~cm}^{-1}$ at $1 \mathrm{THz}$ ) [17]. We note that the use of the IRXCAM-384THz camera for general pulse diagnostics involves illuminating the sensor without the accompanying optical lenses. Bare metallic reflective OAP mirrors are preferred to produce an image at the sensor position, especially when a broadband $\mathrm{THz}$ pulse source is used. Here, the focusing conditions were similar for both tests using OAPs. 
(a)

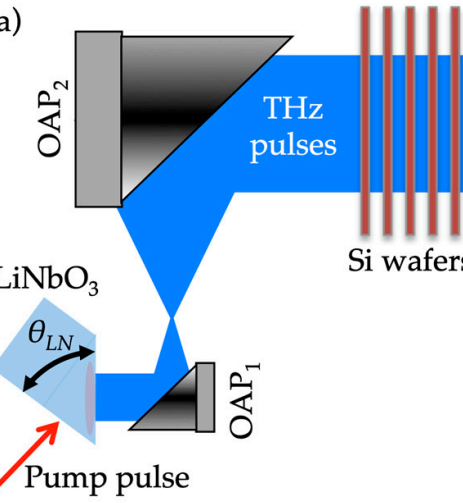

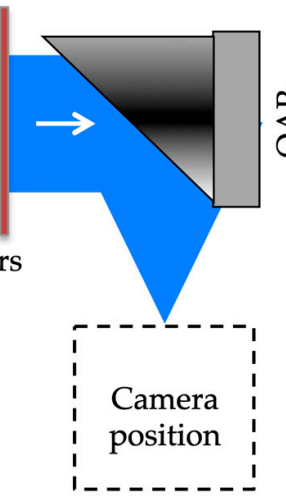

(b)

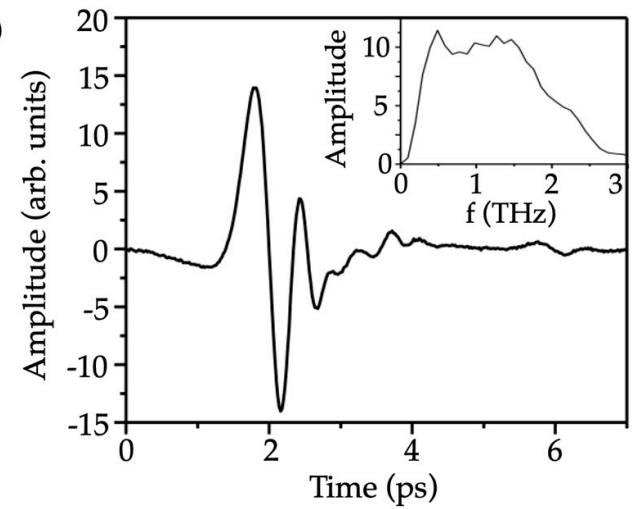

Figure 1. (a) The experimental setup using the tilted-pulse-front pumping scheme for intense terahertz $\mathrm{THz}$ pulse generation, with a tilted angle of $\theta_{\mathrm{LN}}=63^{\circ}$ in a $1.3 \mathrm{~mol} \% \mathrm{MgO}$-doped stoichiometric $\mathrm{LiNbO}_{3}(\mathrm{LN})$ crystal growth by Oxide Corp. The off-axis parabolas OAP1, OAP2, and OAP3 have focal lengths of $10 \mathrm{~mm}, 100 \mathrm{~mm}$ and $100 \mathrm{~mm}$, respectively. (b) Shows the THz pulse with its Fourier transform in inset.

(a)

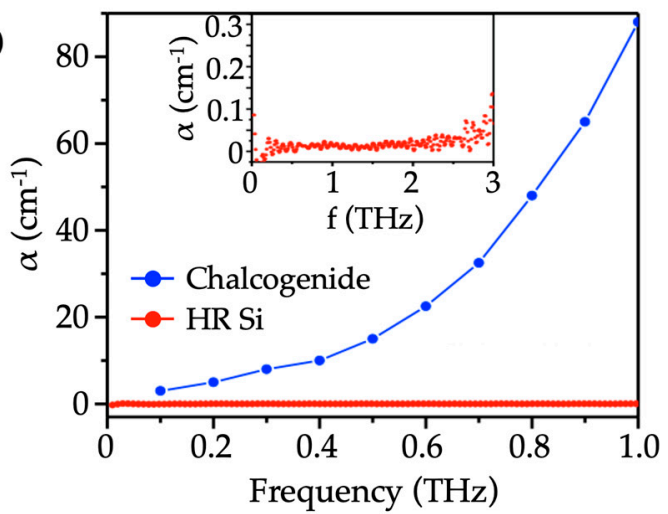

(b)

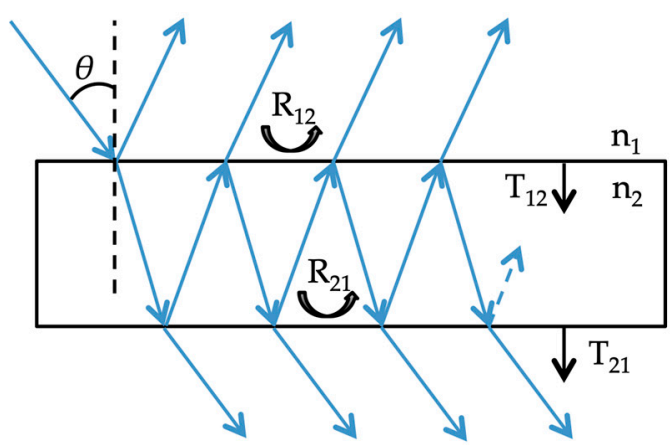

Figure 2. (a) Shows the absorption coefficients of chalcogenide glass [17] and a high resistivity undoped silicon wafer as a function of $\mathrm{THz}$ frequency, respectively. (b) Transmission and reflection geometry inside a silicon wafer.

To attenuate the $\mathrm{THz}$ beam, several high resistivity silicon wafers were inserted in the collimated beam path, as illustrated in Figure 1a. Since the THz absorption in intrinsic silicon is negligible, the transmittance $(\mathrm{T})$ and reflectance $(\mathrm{R})$ of a THz beam depends solely on the Fresnel losses. The situation is illustrated in Figure $2 b$, with the ambient refractive index $\left(n_{1}\right)$, the silicon refractive index $\left(\mathrm{n}_{2}\right)$ and an incidence angle $(\theta)$.

The transmittance $\mathrm{T}_{12}$ denotes the transmittance through the interface, when $\mathrm{THz}$ light comes from medium 1 and passes into medium 2 . The primarily transmitted light is again transmitted into the "third medium", which in our case is identical to the first one (i.e., air) with transmittance $\mathrm{T}_{21}$. At both interfaces, a certain fraction of the THz beam might be reflected, $R_{12}$ at the first surface and $R_{21}$ at the second surface, and so on. The reflectance and the transmittance can be calculated by multiplying the single contribution of the primarily reflected (or transmitted) waves.

By considering $\mathrm{N}$ wafers, the reflectance and transmittance at normal incidence after the ith wafer are given by [19]:

$$
\begin{gathered}
R=\prod_{i=1}^{N} \frac{2 R_{12}}{\left(1+R_{12}\right)}\left(\frac{n_{2}}{n_{1}}|t|^{2}\right)^{(i-1)} \\
T=\left(\frac{n_{2}}{n_{1}}|t|^{2}\right)^{i}
\end{gathered}
$$


with the Fresnel coefficients:

$$
\begin{gathered}
R 12=|r|^{2}=\left(\frac{n_{1}-n_{2}}{n_{2}+n_{1}}\right)^{2} \\
|t|^{2}=\left(\frac{2 n_{1}^{2}}{\left(n_{1}^{2}+n_{2}^{2}\right)}\right)
\end{gathered}
$$

For one silicon wafer, using the refractive index of air $\mathrm{n}_{1}=1$ and the refractive index of silicon $\mathrm{n}_{2}=3.418$ we found $\mathrm{R}=46.1 \%$ and $\mathrm{T}=53.9 \%$ of the total average power. By example, for five silicon wafers and taking into account the multiple reflections and transmissions inside the wafers, the following relation can be used [19]:

$$
T=\left[T_{12} T_{21}+T_{12} R_{21}^{2} T_{21}+T_{12} R_{21}^{4} T_{21}+\ldots\right]^{5}=\left[\frac{1-R_{12}}{1+R_{12}}\right]^{5}
$$

for such case, a transmission of $\mathrm{T}=4.55 \%$ is estimated, which is approximately $135 \mu \mathrm{W}$ in average $\mathrm{THz}$ power.

In the case of chalcogenide glass, its refractive index and absorption coefficient are $\mathrm{n}_{2}=3.5$ and $\alpha=88 \mathrm{~cm}^{-1}$ at $1 \mathrm{THz}$ [17], respectively. Undeniably, in comparison with silicon wafers, the losses in chalcogenide glass at $1 \mathrm{THz}$ are dominated by its large absorption coefficient. For example, the absorbance $\mathrm{A}$ can be expressed in $\mathrm{dB} / \mathrm{cm}$ as a function of distance $\mathrm{x}$ using this simple relation:

$$
A=\log \left(e^{\alpha x}\right)
$$

and at $1 \mathrm{THz}$, adding to its Fresnel losses, a high absorption factor of $38 \mathrm{~dB} / \mathrm{cm}$ in the chalcogenide lens prevents for a good $\mathrm{THz}$ transmission.

\section{Results and Discussion}

To compare both camera performances, imaging of a focused $\mathrm{THz}$ pulse spot and noise equivalent power (NEP) measurement was made. In Figure $3 \mathrm{a}-\mathrm{c}$, the results of the spot size measurements using the Compact Pro and INO cameras are shown. For the Compact Pro demonstration, five silicon wafers were required in the $\mathrm{THz}$ beam path to avoid its intensity saturation, which corresponds to about $135 \mu \mathrm{W}$ of total average $\mathrm{THz}$ power (see Figure $3 \mathrm{~d}$ for a measurement with four silicon wafers). We note that the TPFP scheme is ideally suited to investigate the generation of $\mathrm{THz}$ radiation without interference from the NIR pumping beam at the detector position. The non-collinear phase matching geometry ensures the pump beam (i.e., at $800 \mathrm{~nm}$ ) is geometrically separated from the emitted $\mathrm{THz}$ beam after the lithium niobate emitter crystal, as depicted in Figure 1a.

In Figure 3a are the acquisitions of the THz spot image (i), the background image (ii) and (iii) the result of (i)-(ii), which is a background free $\mathrm{THz}$ image. Only one acquisition frame is used for each image, i.e., without averaging, which is the worst-case scenario for such measurement. In comparison for the IRXCAM-384THz camera, 4 silicon wafers and a chopper were used to perform dynamic background subtraction detection of the THz spot size. Since the attenuation of one silicon wafer corresponds to $53.9 \%$ in average power (as previously obtained with Equation (4)), we have considered the attenuation factor from the chopper to be equivalent to one silicon wafer, i.e., cutting in half $(50 \%)$ the $\mathrm{THz}$ power reaching the sensor. Thus, similar $\mathrm{THz}$ average power was used for both imaging demonstrations. Figure $3 \mathrm{~b}$ shows the result of the same $\mathrm{THz}$ spot size at the sensor position measured by the IRXCAM-384THz camera using dynamic background subtraction detection method, which is definitively the best-case scenario.

In Figure 3c, the profiles of the THz spot size for both cameras are presented. Strikingly, the same $460 \mu \mathrm{m}$ in full width at half maximum (FWHM) THz beam spot size is measured. This information is crucial to confirm the origin of the detected signal. Especially, for a pulse centered at a frequency of $1 \mathrm{THz}$, diffraction limited THz beam spot size should be around $300 \mu \mathrm{m}$ FWHM, or greater [14]. 
Notice that for diffraction limited near infrared (NIR) signal (e.g., at $800 \mathrm{~nm}$ ), the FWHM of such a Gaussian beam would be in the order of micron size at the focus position-which clearly rules out the possibility of NIR detection being here.

(a)
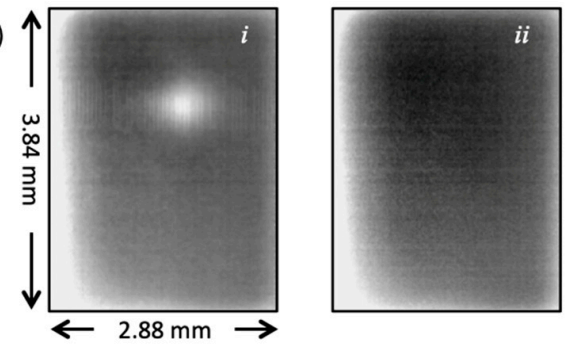

(b)

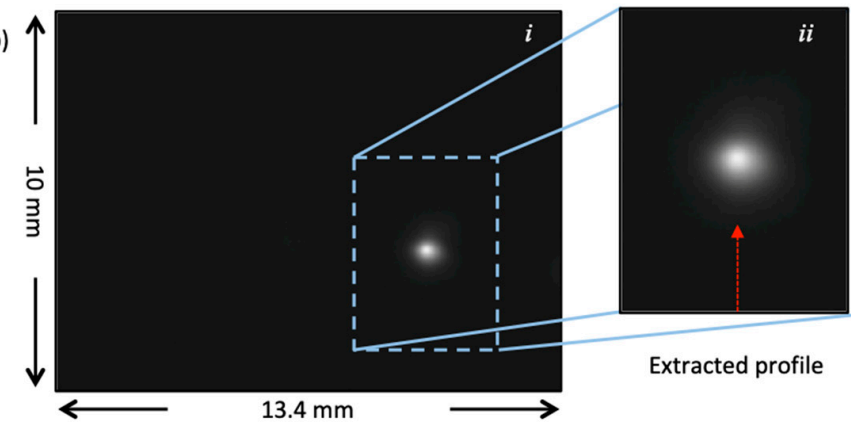

Extracted profile

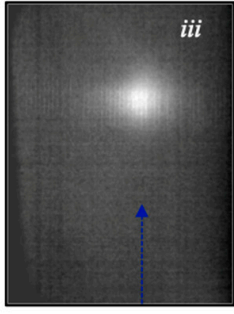

$i i$

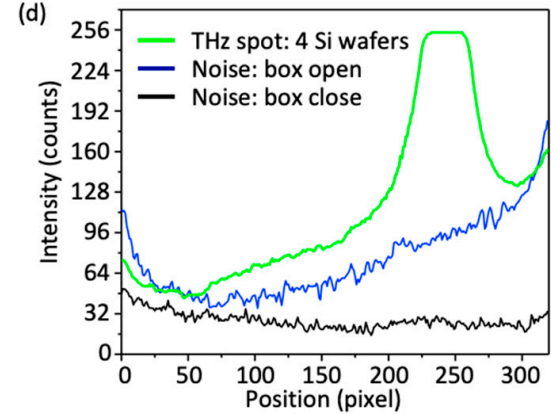

Figure 3. (a) Compact Pro camera result of the THz beam spot imaging at the focus position after $5 \mathrm{Si}$ wafers of attenuation (i) with its background information (ii) and the resulting background free image (iii). (b) IRXCAM-384THz camera result of the same THz beam spot at the focus position using dynamic background subtraction function (i) with an extracted zone corresponding to the same sensor size as the Compact Pro camera. (c) Cross-section intensity profile of THz spot size obtained for both cameras. (d) Recorded background level using 8 bits resolution (limited by the software application) for the Compact Pro in a normal condition (i.e., box closed and chalcogenide lens) and after removing the chalcogenide lens and its framing box (i.e., for THz imaging capability). The profile of a saturated $\mathrm{THz}$ beam spot from the Compact Pro camera with $4 \mathrm{Si}$ wafers of attenuation is also presented.

When comparing the results between the two cameras, a higher background noise level is observed for the Compact Pro camera. This remains the main drawback of recording the background information in a separated acquisition. Especially for this THz demonstration, we decided to completely open the small framing box of the Compact Pro together with the chalcogenide lens. Only the bare circuit board with its sensor remained connected to our iPhone. In such condition, the surrounding heat is not well blocked by the tiny mechanical chopper in front of the VO2 sensor. As it can be found in Figure 3d, a higher background level appears when the chalcogenide lens is removed with the framing box. Nevertheless, both cameras read the same information, i.e., a full width at half maximum (FWHM) spot size of $\sim 460 \mu \mathrm{m}$, which clearly indicates the ability of the Compact Pro thermal camera to read in the $100 \mathrm{GHz}$ to $2.5 \mathrm{THz}$ frequency range, as expected from the inset of Figure $1 \mathrm{~b}$. To complement the $\mathrm{THz}$ imaging demonstration, we present in information supplementary information S1 a video of a live measurement of the THz spot image taken with an iPhone 6 and a Compact Pro camera. This recording was made by manually moving the camera in the $\mathrm{THz}$ focus plane.

To have a fair comparison with the scientific camera from INO, we have evaluated the noise equivalent power (NEP) of the Compact Pro camera following the same procedure as demonstrated in recent works [20-22]. To do so, we used the signal level obtained from the integration of counts within the sensing area (e.g., an area covered by $100 \times 100$ pixels in our case). As mentioned previously, using a pyroelectric detector from Gentec, model THZ51-BL-BNC-D0, a THz energy of $\sim 3 \mu \mathrm{J}$ at $1 \mathrm{kHz}$ repetition rate was measured, which corresponds to an average power of $\sim 135 \mu \mathrm{W}$ after attenuation 
by five silicon wafers. The noise level was calculated from the standard deviation of the fluctuations at each pixel. In Figure 4, we show an example of the noise fluctuations for a single pixel and for a $100 \times 100$ pixels area as a function of time (i.e., while targeting a uniform heat object consisting of a metallic cover). In this measurement, an acquisition frame rate of $9 \mathrm{~Hz}$ is estimated from the 90 images recorded in 10 seconds (i.e., also expected from the manufacturer specifications). It is clear from Figure 4 that the 8 bits resolution of the Compact Pro is not enough to resolve the noise fluctuations. Mainly, the thermal drift of the device is observed with sudden jumps whenever the mechanical shutter is activated (as seen by the averaging behaviors from the $100 \times 100$ pixels area). It is also clear from Figure 4 that the single pixel noise reading is numerically clipped as a function of time. Thus, our standard deviation evaluation does not provide the real limitation in sensitivity of Compact Pro camera. Nevertheless, from these curves, we estimated a conservative standard deviation of the fluctuations to be in the order of $0.5 \%$ of the full dynamic range.

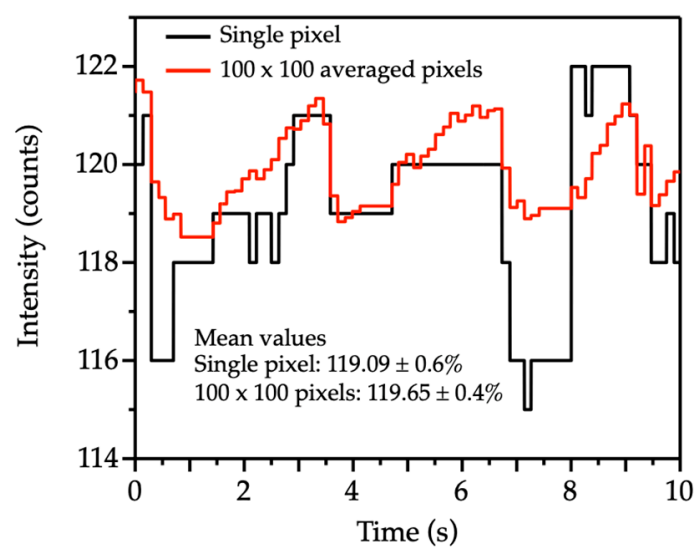

Figure 4. Noise fluctuations as a function of time for one pixel and an area of $100 \times 100$ pixels. A metallic plate covers the camera sensor.

For a recorded area of $100 \times 100$ pixels, we found an integrated number of counts of 1,181,598, which corresponds to a $135 \mu \mathrm{W}$ in $\mathrm{THz}$ power reading divided by the camera frame rate of $9 \mathrm{~Hz}$. After subtracting the background information, a signal to noise ratio of 140 was estimated. This measurement indicates a NEP of about $100 \mathrm{nW}$ for the Compact Pro camera in the 0.1 to $2.5 \mathrm{THz}$ frequency range. This number is well above the $<100 \mathrm{pW}$ of the IRXCAM-384THz camera from INO [21], as expected for a scientific camera. Nonetheless, the Compact Pro camera offers a functional and affordable imaging capture device for the $\mathrm{THz}$ frequency range. A simple additional modification, such as changing the mechanical chopper to a synchronous background subtraction, would drastically improve its scientific value. Moreover, adding the capability to save the data in the raw format of the native 14 bits analog to digital converter would also bring this camera to another level, without large expense. In such a case, the signal resolution would be improved by a factor 64 , and at the same time, the minimum detectable signal or NEP would be improved accordingly.

\section{Conclusions}

We have demonstrated a low cost $\mathrm{THz}$ imaging camera with a price that is $1 / 100$ th that of commercially available $\mathrm{THz}$ cameras. We point out that by simply removing the chalcogenide lens from the Compact Pro thermal imaging camera from Seek Thermal it functions as a low-cost THz camera. Two modifications would greatly benefit the scientific or industrial use of the Compact pro; saving data in the native 14 bits format and adding an external trigger function for the dynamic background subtraction detection method. Nevertheless, this camera is ready to be used for the mandatory alignment of typical $\mathrm{THz}$ sources based on the tilted-pulse front excitation in lithium niobate. 
Supplementary Materials: Supplementary materials can be accessed at http://www.mdpi.com/2076-3417/9/12/ 2531/s1. Video S1: Compact Pro imaging using an iPhone device of a THz beam at the focus position. An estimated average power of $135 \mu \mathrm{W}$ in the $\mathrm{THz}$ frequency band ranging from $100 \mathrm{GHz}$ to $3 \mathrm{THz}$.

Author Contributions: Conceptualization, F.B.; methodology, F.B., J.E.N. and D.M.; validation, F.B., J.E.N., and D.G.C.; resources, F.B., R.N., and D.G.C.; writing, review and editing, F.B., J.E.N., M.B., R.N., and D.G.C.; project administration, F.B. and R.N.

Acknowledgments: F.B. gratefully acknowledges financial support from NSERC, FRQNT and the ÉTS-Chaire in THz optoelectronics. D.C. would like to acknowledge financial support from NSERC.

Conflicts of Interest: The authors declare no conflict of interest.

\section{References}

1. Guerboukha, H.; Nallappan, K.; Skorobogatiy, M. Toward real-time terahertz imaging. Adv. Opt. Photonics 2018, 10, 843-938. [CrossRef]

2. Mittleman, D.M. Twenty year of terahertz imaging. Opt. Express 2018, 26, 9417-9431. [CrossRef] [PubMed]

3. Kawase, K.; Ogawa, Y.; Watanabe, Y.; Hiroyuki, I. Non-destructive terahertz imaging of illicit drugs using spectral fingerprints. Opt. Express 2003, 11, 2549-2554. [CrossRef] [PubMed]

4. Karpowicz, N.; Zhong, H.; Zhang, C.; Lin, K.-I.; Hwang, J.-S.; Xu, J.; Zhang, X.-C. Compact continuous-wave subterahertz system for inspection applications. Appl. Phys. Lett. 2005, 86, 054105. [CrossRef]

5. Jansen, C.; Wietzke, S.; Peters, O.; Scheller, M.; Vieweg, N.; Salhi, M.; Krumbholz, N.; Jördens, C.; Hochrein, T.; Koch, M. Terahertz imaging: Applications and perspectives. Appl. Opt. 2010, 49, E48-E57. [CrossRef] [PubMed]

6. Darmo, J.; Tamosiunas, V.; Fasching, G.; Kröll, J.; Unterrainer, K.; Beck, M.; Giovannini, M.; Faist, J.; Kremser, C.; Debbage, P. Imaging with a terahertz quantum cascade laser. Opt. Express 2003, 11, 2549-2554. [CrossRef] [PubMed]

7. Russe, I.-S.; Brock, D.; Knop, K.; Kleinebudde, P.; Zeitler, J. Validation of terahertz coating thickness measurements using X-ray microtomography. Mol. Pharm. 2012, 9, 3551-3559. [CrossRef] [PubMed]

8. Fukunaga, K.; Hosako, I. Innovative non-invasive analsyis techniques for cultural heritage using terahertz technology. Comptes Rendus Phys. 2010, 11, 519-526. [CrossRef]

9. Grossman, E.; Dieltein, C.; Ala-Laurinaho, J.; Leivo, M.; Gronberg, L.; Gronholm, M.; Lappalainen, P.; Rautiainen, A.; Luukanen, A. Passive terahertz camera for standoff security screening. Appl. Opt. 2010, 49, E106-E120. [CrossRef] [PubMed]

10. Ok, G.; Park, K.; Kim, H.J.; Chun, H.S.; Choi, S.-W. High speed terahertz imaging toward food quality inspection. Appl. Opt. 2014, 53, 1406-1412. [CrossRef] [PubMed]

11. Blanchard, F.; Razzari, L.; Bandulet, H.-C.; Sharma, G.; Morandotti, R.; Kieffer, J.-C.; Ozaki, T.; Reid, M.; Tiedje, H.F.; Haugen, H.K.; et al. Generation of $1.5 \mu \mathrm{J}$ single-cycle terahertz pulses by optical rectification from a large aperture ZnTe crystal. Opt. Express 2007, 15, 13212-13220. [CrossRef] [PubMed]

12. Oda, N.; Yoneyama, H.; Sasaki, T.; Sano, M.; Kurashina, S.; Hosako, I.; Sekine, N.; Sudoh, T.; Irie, T. Detection of terahertz radiation from quantum cascade laser using vanadium oxide microbolometer focal plane arrays. Proc. SPIE 2008, 6940, 69402Y.

13. Bolduc, M.; Marchese, L.; Tremblay, B.; Doucet, M.; Terroux, M.; Oulachgar, H.; Le Noc, L.; Alain, C.; Jerominek, H.; Bergeron, A. Video-rate THz imaging using a microbolometer-based camera. In Proceedings of the 35th International Conference on Infrared Millimeter and Terahertz Waves (IRMMW-THz), Rome, Italy, 5-10 September 2010; Volume 1.

14. Hirori, H.; Doi, A.; Blanchard, F.; Tanaka, K. Single-cycle terahertz pulses with amplitudes exceeding $1 \mathrm{MV} / \mathrm{cm}$ generated by optical rectification in $\mathrm{LiNbO}_{3}$. Appl. Phys. Lett. 2011, 98, 091106. [CrossRef]

15. Hafez, H.A.; Chai, X.; Ibrahim, A.; Mondal, S.; Férachou, D.; Ropagnol, X.; Ozaki, T. Intense terahertz radiation and their applications. J. Opt. 2016, 18, 093004. [CrossRef]

16. Available online: https://support.thermal.com/hc/en-us/articles/115001285630-What-is-Thermal-Imaging(accessed on 3 April 2019).

17. Ravagli, A.; Naftaly, M.; Craig, C.; Weatherby, E.; Hewak, D.W. Dielectric and structural characterization of chalcogenide glasses via terahertz time-domain spectroscopy. Opt. Mater. 2017, 69, 339-343. [CrossRef] 
18. Dai, J.; Zhang, J.; Zhang, W.; Grischkowsky, D. Terahertz time-domain spectroscopy characterization of the far-infrared absorption and index of refraction of high-resistivity, float-zone silicon. J. Opt. Soc. Am. B 2004, 21, 1379-1386. [CrossRef]

19. Stenzel, O. The Physics of Thin Film Optical Spectra-An Introduction; Springer: Berlin, Germany, 2005.

20. Oda, N. Uncooled bolometer-type terahertz focal plane array and camera for real-time imaging. C. R. Phys. 2010, 11, 496-509. [CrossRef]

21. Bolduc, M.; Terroux, M.; Tremblay, B.; Marchese, L.; Savard, E.; Doucet, M.; Oulachgar, H.; Alain, C.; Jerominek, H.; Bergeron, A. Noise-equivalent power characterization of an uncooled microbolometer-based THz imaging camera. Proc. SPIE 2011, 8023, 80230C-1.

22. Hack, E.; Valzania, L.; Gäumann, G.; Shalaby, M.; Hauri, C.P.; Zolliker, P. Comparison of thermal detector arrays for off-axis THz holography and real-time imaging. Sensors 2016, 16, 221. [CrossRef] [PubMed]

(C) 2019 by the authors. Licensee MDPI, Basel, Switzerland. This article is an open access article distributed under the terms and conditions of the Creative Commons Attribution (CC BY) license (http://creativecommons.org/licenses/by/4.0/). 